\title{
A Methodology for Defining Working Healthcare Ontologies
}

\author{
Emine Sezer (Corresponding author) \\ Department of Computer Engineering, Faculty of Engineering, Ege University, Izmir, Turkey \\ E-mail: emine.sezer@ege.edu.tr \\ Murat Osman Unalir \\ Department of Computer Engineering, Faculty of Engineering, Ege University, Izmir, Turkey \\ E-mail: murat.osman.unalir@ege.edu.tr \\ Ozgu Can \\ Department of Computer Engineering, Faculty of Engineering, Ege University, Izmir, Turkey \\ E-mail: ozgu.can@ege.edu.tr \\ Okan Bursa \\ Department of Computer Engineering, Faculty of Engineering, Ege University, Izmir, Turkey \\ E-mail: okan.bursa@ege.edu.tr
}

\begin{abstract}
Retrieving the information needed for right clinical decision and searching through a large amount of data are important challenges that health information systems encountered despite the advances in the information systems. In order to reuse these distributed data, the health information standards are developed to enable interoperable health information systems. As the standards are far from the necessary semantics, the data defined according to these standards are not been efficiently used by machines. Semantic Web technologies provide a common framework to access and process the information by machines with the support of ontologies. The heterogeneous and distributed nature of health data makes it a very suitable candidate to define health domain specific ontologies. In this work, a methodology for defining health ontologies, which should work as information base of a health information system as the next stage, is proposed to ensure interoperability and reuse between health information systems with the support of health information standards. Clinical Biochemistry Laboratory Ontology (CBLO) is given as a use case to model information of laboratory tests realized in clinical biochemistry laboratory that are a sub-domain of medical laboratory tests. In addition, a weighted evaluation for the proposed methodology is presented.
\end{abstract}

Keywords: Healthcare ontologies, Ontology, Methodology, Semantic Web, Interoperability, Health Information Standards, Knowledge Representation.

DOI: $10.7176 / \mathrm{JSTR} / 5-4-15$

\section{Introduction}

Healthcare services, one of the vital requirements of human, continues throughout their life long with the beginning before their births. The health domain, which is very rich in terms of technical terms, is a field where very complex and voluminous data are constantly produced and used with the additional personalized health information. This data is constantly growing, also. Storing, processing and managing of these big health data is one of the important research areas that have been studied in information technologies over the last decade. Another significant research topic is retrieving the accurate and exact information that is required to diagnose a specific health problem [Gesulga et al. 2017; Edinger et al. 2012].

Health data standards are developed to share highly structured and richly meaningful health data that are defined in distributed environments, across systems. Data standards are the principal informatics component necessary for information flow through the health information infrastructure [Erickson et al. 2003; Schulz et al. 2019]. Data modeled with a specific standard can be transmitted between different systems with ensuring that the meaning of the data does not change from system to system, from program to program or from institution to institution. Despite the many standards being defined in the health field, 
one of the barriers to electronic health records is interoperability [Kruse et al. 2016; Fung and Bodenreider 2019]. This is because to meet specific clinical needs so many health standards are developed. Besides the interoperability problem, the other important constraint is that the health data standards do not contain the semantics of health data, which can be easily processed by the computers and make reasoning on it.

Ontology-based information querying and retrieval have proven to be a solution that allow users to navigate through terms and relationships, or to elaborate general or nonspecific questions by using defined semantic relationships between concepts [Schulz et al. 2006]. By using Semantic Web technologies in health information systems, interoperability and reusability of health data from different sources can be ensured.

Semantic Web is defined as the extension of the current web where information is given in a well-defined meaning and leads to a better collaboration between computers and humans [Berners-Lee et al. 2001]. As a result of using Semantic Web technologies, data processing will be faster and more accurate, while automation of the most of the healthcare services is achieved. Thus, sharing and interoperability will cause big data. In health information systems, that leads to better information retrieval, inferencing new information from unexploited data, enhanced interoperability between institutions and better health services for the patients [Kolias et al. 2014]. Consequently, it is expected that the diagnosis and treatment processes could accelerate while the cost will decrease.

To enable the health information to be used in the diagnosis and treatment processes by machines, the health domain specific ontologies have been developed in recent years. In this article, an ontology development methodology is proposed to develop healthcare ontologies which can be used as the information base for health information systems. The main purpose of the proposed methodology is to ensure the interoperability between the health information systems which are developed independently and are used by different intuitions. Clinical Biochemistry Laboratory Ontology (CBLO) is used as the use case for better explanation of the proposed methodology.

The organization of the paper is as follows: Section 2 presents the related work. Section 3 describes the proposed methodology for defining healthcare ontologies step by step. The evaluation of the proposed methodology is given in Section 4. Finally, Section 5 contributes and outlines the direction of the future work.

\section{Literature Review}

Software systems developed for healthcare applications focus on gathering clinical data, connecting to clinical knowledge bases, querying and retrieving the required information as well as data clustering and exchanging. Data are usually recorded at different health centers, on different times and in different ways. The primary purpose in health information systems is storing the health records belonging to the patients. The target with EHR (Electronic Health (care) Record) is to provide reliable clinical data entry that meets the communication requirements between machines and human [Ceusters and De Moor 2005]. For this purpose, emphasis has been given to clinical coding and standardization. Because the required data cannot be fully defined by these systems, the studies have been focused the development of the domain specific ontologies in particular to standardize the domain specific technical terms.

Nowadays, there are lots of ontologies and terminologies, like FMA (Foundation Model of Anatomy) [FMA 2019], RadLex (Radiology Lexicon) [RadLex 2019], UMLS [UMLS 2019], SNOMED-CT (Systemic Nomenclature of Medicine-Clinical Terms) [SNOMED-CT 2019], ICD (International Classification of Diseases) [ICD 2019], which are developed for different purposes. The common property of these terminologies is that they contain quite a few concepts. It is a difficult and also time consuming process to search within these comprehensive terminologies in order to find the appropriate concept. For this reason, in order to use only the required concepts, the defined more general ontologies are customized for sub-health domains for specific clinics such as imaging, medical testing and etc.

These customizations have been expanded to meet the requirements of that specific area, and ontologies have been developed to identify relevant clinical units. Ontologies such as NeuroFMA [Nolan et al. 2014], MEDICO [Seifert et al. 2010] and SEMIA [Dorn and Jager 2004] are modeled on the basis of FMA ontology. FMA-RADLEX [Mejino et al. 2008] is another ontology developed by reusing relevant concepts from both RadLex and FMA ontologies.

SNOMED-CT [Stearns et al. 2001] offers a multi-lingual health terminology, which is commonly used in the world for defining the clinical data. It describes the health information used in medical studies to improve the health services. SNOMED-CT has a hierarchical structure with 324,129 concepts and 152 object features.

ICD is defined as the international disease classification standard used for all general epidemiological, health cost management and clinical services. ICD-10 is the current version of the ICD-9 classification

147 | $P$ a g e

www.iiste.org 
that is used between 1979 and 1998 . The current version of ICD-10 has 12,445 concepts with only one object property. ICD-10 CM [Del Mar Roldán-Garcia et al. 2016] and ICD-10 PCS [ICD-10 PCS 2019] ontologies are also developed on the basis of ICD-10 ontology for specific clinical domains. The beta version of ICD-11 [Tudorache et al. 2013] is issued in 2017.

Medical laboratories are critical components for diagnosing and following the treatment in clinical operations. The information produced in laboratories can be in different forms like numbers, text, graphs, or other images with interpretative data. LIS (Laboratory Information Systems) become critical components to manage the flow of information between health professionals, patients and health institutes. The characteristics that a LIS should have in order to share the produced data in these laboratories is identified [Sepulveda et al. 2013; Tagger 2011; Nyasulu, et al. 2014]. The common recommendation for successful LIS in these studies is the necessity of using Semantic Web technologies to identify the data for interoperability and reuse.

There are many efforts on the use of informatics standards and ontologies in the field of health domain. However, these studies focus on defining a specific domain in healthcare from the point of view of health professionals. It cannot be seen that these studies can be used as information base in the next stage of a living health information systems. However, in developed systems, traditional database applications are used, which are not capable to share health information among different health information systems.

\section{Methodology for Defining Working Health Ontologies}

Health Information Systems are encountered with many challenges, among them the management of an increased amount of data which need to be acquired, stored, processed and presented at the right time and place in an accessible form [Focsa 2016]. Retrieving the information needed for right clinical decision and searching through a large amount of data are outstanding problems despite the advances in the information systems.

The health information standards are developed to solve the interoperability problems in the health information standards. However, the developed standards cannot achieve the complete coverage of the clinical language. Semantic Web technologies provide a common framework to access and process the information by machines with the support of ontologies. The ontologies are used to describe the realworld entities and relationships between these entities in a specific domain. The heterogeneous and distributed nature of health data makes it a very suitable candidate for Semantic Web Applications [Rajbhandari et al. 2012; Burgun et al. 1999]. There have been recently some efforts to use ontologies to describe health information standards [Möller et al. 2010].

The usage of ontologies should not be limited to just defining the domain. The use of the developed ontologies as a knowledge base of an information system will enable the sharing and reusability of the information defined in that information system with other systems. At the same time, inferencing and reasoning support provided by Semantic Web technologies can also be used to obtain new information these ontologies are called now as working ontologies. There are lots of works to define methodology for building ontologies like [Uschold and Gruninger 1996; Noy et al. 2001; Corcho et al. 2003; Cristani, and Cuel 2005]. As defined in those works, there is not only one true way to define an ontology. The methodology proposed in this study aims to help ontology developers by defining the steps needed to build ontologies in the health domain, which can be used as information base in health information systems by ensuring the interoperability and reuse. The proposed methodology is illustrated in Figure 1. 


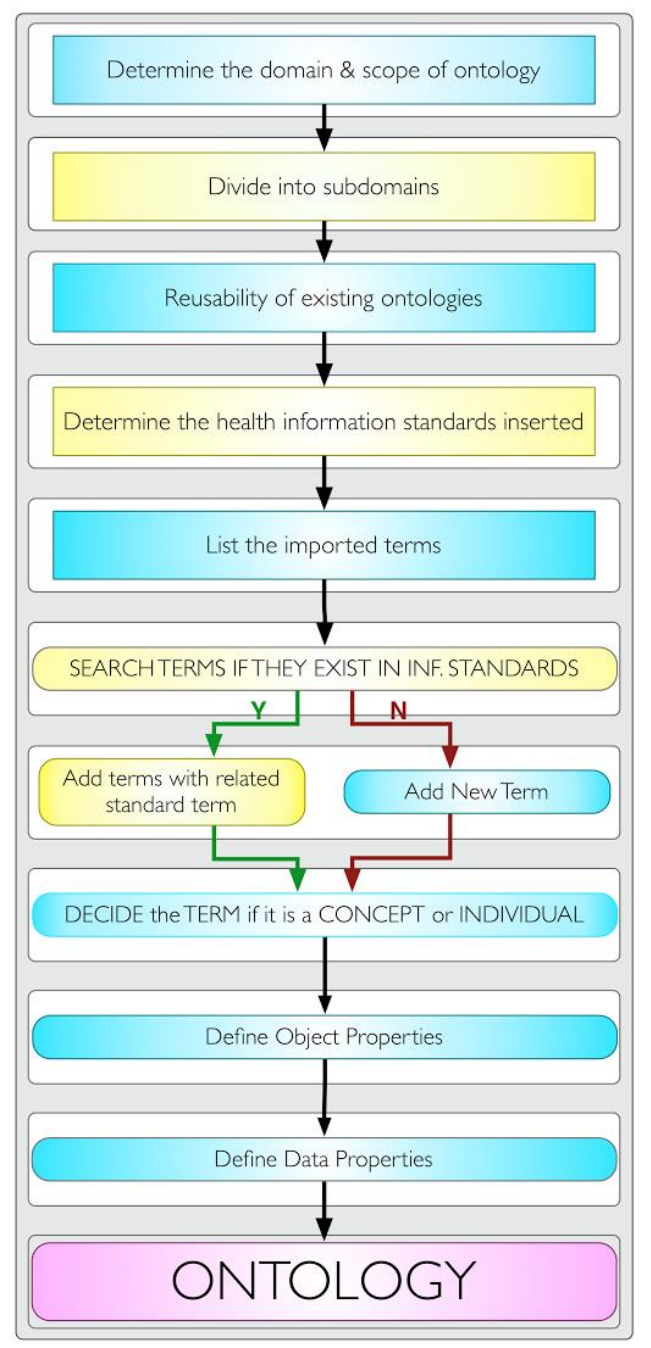

Figure 1. The methodology to develop interoperable health ontologies by using Healthcare Information Standards.

Determining the domain and scope of ontology: Determining the domain and scope of ontology is always proposed as the first step by ontology building methodologies. Determining the scope of the ontology for the healthcare domain is a critical task for the manageability of the health information system where the ontology will be used. The scope generally depends on the view of ontology engineer who builds the ontology with the domain expert. The main aim should be considering the purpose of the usage of the developed ontology whether it is used as pure information base to track the patients' health data or also as a decision support system to give advices to patients as well as health professionals [Shridevi et al. 2018]. First of all, when a patient is admitted to a clinic with any complaint, all medical data related to the patient should be accessed by the doctor. New information can be provided to guide the patient or a doctor for giving decision about the complaints in the light of the new clinical findings by using machine learning or inferencing. Therefore, the scope of ontology to be developed should be clearly defined.

Dividing into subdomains: When developed ontologies and terminologies are examined, it can be seen that their scopes are very broad so that they include lots of terms as in SNOMED-CT. To manage and maintain ontology easily and effectively, we recommend that dividing health domain in clinical level to define more useful and functional ontologies. For example, laboratory tests include biochemistry, endocrinology, microbiology, radiology etc. examinations. Defining a laboratory ontology for all these clinical units would have lots of concepts which results difficulties to manage the information. Therefore, 
building an ontology for each clinical unit will be easier, more functional, more manageable and easily maintainable to describe that domain. As a use case, Clinical Biochemistry Laboratory Ontology (CBLO) is developed to examine this proposed methodology.

Reusing the existing ontologies: As in many ontology development methodologies, reusability of existing ontologies has to be considered for interoperability [Azarm and Peyton 2018)]. There are lots of ontologies that are developed for defining the healthcare domain. To develop working healthcare ontologies, selecting the right existing ontology to be reused is a challenging task. In order to simplify this challenge, we propose inserting the health information standards for interoperability as the next task.

Determining health information standards to be inserted: In addition to reusing existing ontologies, we also recommend that the health information standards that are widely used in the world should be inserted to building ontologies. In this way, it will be possible to exchange information through other information systems or mappers using these standards. Importing all of the terms from the terminologies is not recommended since the unused concepts make the ontology unwieldy. SNOMED-CT, ICD-10 and ICD11 standards are selected to insert in developed CBLO ontology.

Listing important terms: Listing important terms are recommended to be done with health professionals. Health information systems are multidisciplinary field where professionals from both health and computer domains have to work together.

Defining the concepts, individuals and hierarchy: After listing the terms, the ontology engineers can start to develop ontology by defining concepts considering the proper hierarchical levels. The hierarchical structure is important to make reasoning and inferencing.

The most challenging point in building ontology in health domain is deciding a particular concept is a class or an individual instance. When the developed ontologies and terminologies are examined, it is seen that all the terms related to health domain are defined as classes and there is not any individual instance defined. In living information systems, the maintenance should be an important case. To facilitate the maintenance, it is necessary to make the number of classes as stable as possible and to make sufficient definitions to insert individuals to the information base during the operation of the health information system.

Defining object properties: Defining object properties between concepts is another important step to navigate through concepts. In CBLO, we defined an object property as hasMedicalinformationstandard, which has three sub-object properties as hasSNOMEDCTConceptID, hasICD10Code and hasICD11Code. These properties have ranges SNOMEDCTConceptID, ICD1OCode and ICD11Code, which are subclasses of concept Medical Informationstandard, respectively. These definitions are shown in Figure 2.

Defining data properties: The attributes of a concept's individuals and the range values that these attributes can take are defined in this step. Considering that ontology will work as an information base of a health information system, both object properties and data properties must be defined in such a carefully way as not to cause any conflicts.

For a use case, Doctor01 suspects from cholestasis after listening the complaints of his patient, Patient01. For this reason, he requests clinical biochemistry tests to measure the substances ALT, AST, ALP, Albumin, Total Bilirubin and Prothrombin Time. In bile duct obstruction, the level of ALP is four times higher than the normal value while no significant abnormality is seen in other tests. The test results of Patient 01 and the ontological representation of relations are illustrated in Figure 3. 


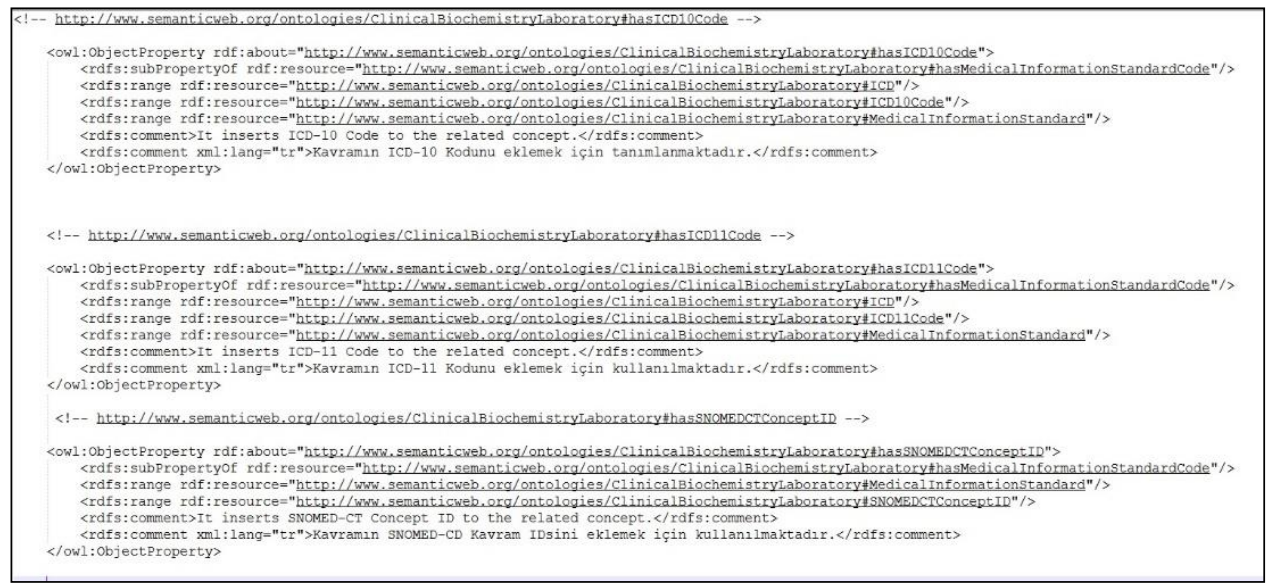

Figure 2. The definitions of object properties for Medical Information Standards.

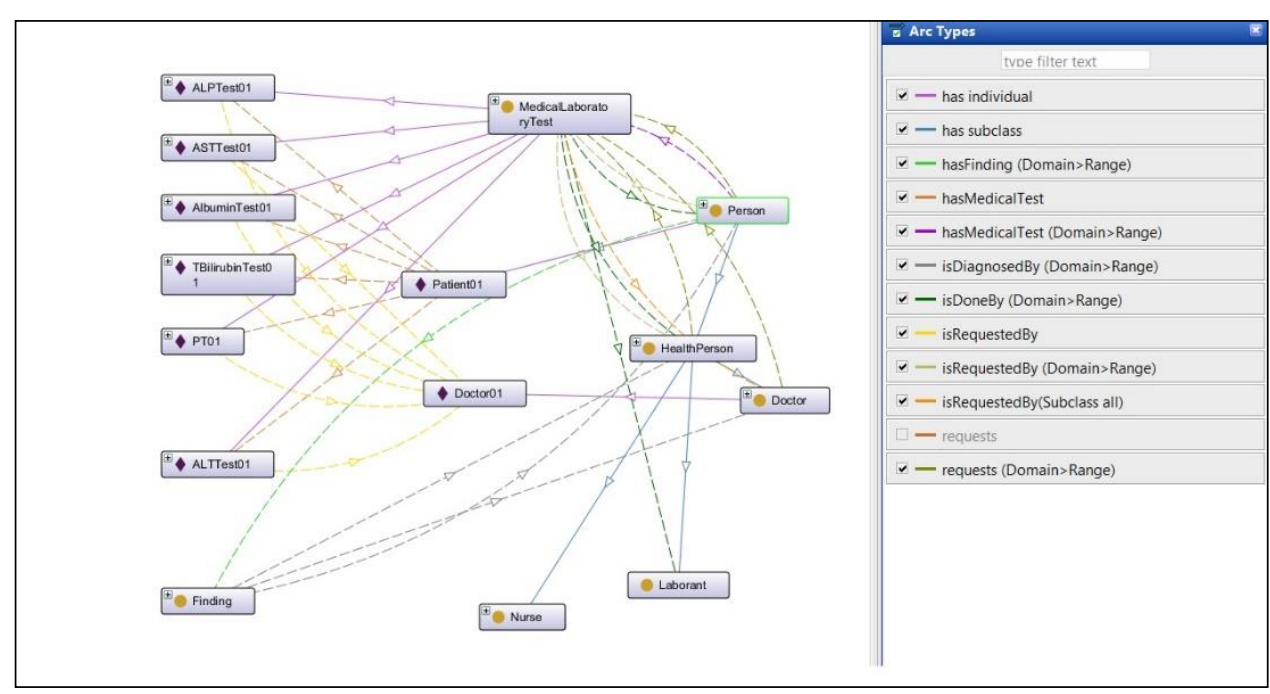

Figure 3. The representation of relations between defined individuals. 
According to these knowledge, a rule can be defined by using concepts, the concepts' instances, their object properties and data properties in developed ontology. When the rule is processed, it is inferred that Patient01 is likely to have cholestasis finding as shown in Figure 4.

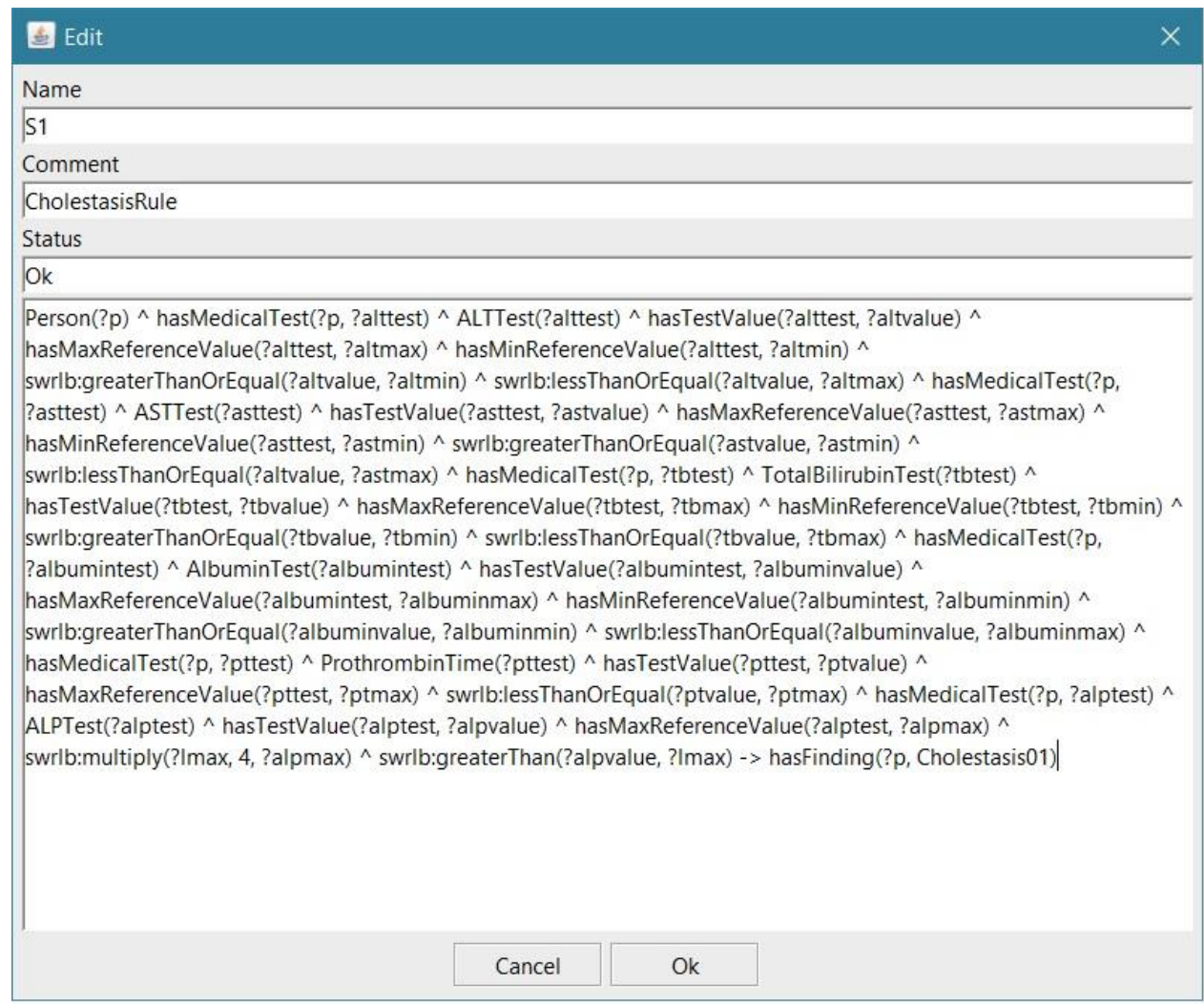

Figure 4. The cholestasis rule according to the results of clinical biomedical laboratory test.

\section{Evaluation of Methodology}

The quality and effectiveness of semantic web applications is based on the quality and effectiveness of the ontology the application uses. The methodology used to create effective ontologies is also an important point in this context. There are many ontology development methodologies in literature and there is no right one [Gómez-Pérez et al. 2004].

To evaluate our proposed methodology, the weighted evaluation for ontology building methods [Hakkarainen et al. 2005] is used. The evaluation method consists of seven semiotic categories $\left(s_{I}\right.$ to $\left.s_{7}\right)$ of modelling methodologies described in [Krogstie 1995] and suggests coverage weight $\left(c w\left(s_{n}\right)\right)$ for each criteria with values $-1,1$ and 2 . Each criteria also has importance weight $\left(i w\left(s_{n}\right)\right)$ which is calculated with the Eq. 1 as follows according to the requirements categorized in [Hakkarainen et al. 2005].

$$
i w\left(s_{n}\right)=\left\{\begin{array}{l}
1, \text { if } s_{n} \text { may be satisfied, it is optional, } \\
3, \text { if } s_{n} \text { should be satisfied, it is recommended } \\
5, \text { if } s_{n} \text { must be satisfied, it is essential, }
\end{array}\right.
$$

As a result, a total coverage weight, $T w$, is calculated by using Eq.2.

$$
T w=\sum_{i=1}^{7}\left(c w\left(s_{i}\right) \times i w\left(s_{i}\right)\right)
$$


Weltanschauung $\left(s_{1}\right)$, describes the underlying philosophy or view to the world with three views: the objectivistic view, the constructivistic view, and the mentalistic view [Hakkarainen et al. 2005]. Our proposed methodology is constructivistic because domain assumptions is made explicitly with healthcare professionals and ontology engineers' collaboration. Therefore, the coverage weight is equal to 2 . However, the constructivistic view is not a crucial requirement, so its importance weight is equal to 3.

Coverage in process $\left(s_{2}\right)$ concerns the method's ability to address the development process as planning for changes $\left(s_{2} c_{1}\right)$, single and co-operative development of the ontology $\left(s_{2} c_{2}\right)$, use and operations of ontology $\left(s_{2} c_{3}\right)$, maintaining and evaluation of ontologies $\left(s_{2} c_{4}\right)$ and management of planning, development, operations, and maintenance of ontologies $\left(s_{2} c_{5}\right)$. Our proposed methodology addresses $s_{2} c_{1}, s_{2} c_{2}, s_{2} c_{3}, s_{2} c_{4}$, and $s_{2} c_{5}$ issues, consequently $c w\left(s_{2}\right)$ equals to $2 . i w\left(s_{2}\right)$ has the value of 5 because an ontology building method must be satisfied and essential.

Coverage in product examines the method's ability to operate on one single ontology $\left(s_{3} c_{1}\right)$, a family of related ontologies $\left(s_{3} c_{2}\right)$, a whole portfolio of ontologies in an organization $\left(s_{3} c_{3}\right)$, and a totality of the goals, business process, people and technology used within the organization $\left(s_{3} c_{4}\right)$. Our proposed ontology satisfies $s_{3} c_{1}, s_{3} c_{2}$ and $s_{3} c_{4}$ issues, so that $c w\left(s_{3}\right)$ equals to 2 . $i w\left(s_{3}\right)$ equals to 1 because development of a single ontology in a stand-alone application may be supported.

Reuse of product and process support reuse of ontologies as products or reuse of method as processes according to the six dimensions of reuse by trying to answer the related questions in [Hakkarainen et al. 2005]: reuse by motivation $\left(s_{4} c_{1}\right)$, reuse by substance $\left(s_{4} c_{2}\right)$, reuse by development process $\left(s_{4} c_{3}\right)$, reuse by management mode $\left(s_{4} c_{4}\right)$, reuse by technique $\left(s_{4} c_{5}\right)$, and reuse by intentions $\left(s_{4} c_{6}\right)$. Our proposed methodology aims the reuse to make interoperable healthcare information systems and have answers for all dimensions, therefore $c w\left(s_{4}\right)$ equals to 2 . Because the reuse is critical and the health information standards have to be inserted into the ontology, $i w\left(s_{4}\right)$ equals to 5 .

Stakeholders participation reflects the interests of different actors in the ontology building process: those responsible for developing the method $\left(s_{5} c_{1}\right)$, those with the financial interest $\left(s_{5} c_{2}\right)$, and those having interest in use $\left(s_{5} c_{3}\right)$. Our proposed methodology consists the $s_{5} c_{1}$ and $s_{5} c_{3}$, so $c w\left(s_{5}\right)$ equals to 2 . The ontology building methodology should cover the participants' contribution, $i w\left(s_{5}\right)$ equals to 3 .

Representation of product and process can be informal $\left(s_{6} c_{1}\right)$, semi-formal $\left(s_{6} c_{2}\right)$ or formal $\left(s_{6} c_{3}\right)$. Our proposed methodology is mostly informal $\left(s_{6} c_{1}\right)$. Therefore, $c w\left(s_{6}\right)$ equals to -1 . $i w\left(s_{6}\right)$ has value of 3 .

Maturity is characterized on different level of completion as fully-described $\left(s_{7} c_{1}\right)$, adaptation, navigation and development $\left(s_{7} c_{2}\right)$, used and updated through practical applications $\left(s_{7} c_{3}\right)$, used by many organizations $\left(s_{7} c_{4}\right)$, and altered $\left(s_{7} c_{5}\right)$. The satisfied issues of our propped methodology are $s_{7} c_{1}$ and $s_{7} c_{3}$ with the $c w\left(s_{7}\right)$ value 1 . This criterion should be satisfied, so it has $i w$ value of 3 . Table 1 summarizes the evaluation of the proposed methodology for defining working healthcare ontologies.

Table 1. Evaluation of proposed methodology

\begin{tabular}{lllllllll}
\hline Evaluation criterion & $s_{1}$ & $s_{2}$ & $s_{3}$ & $s_{4}$ & $s_{5}$ & $s_{6}$ & $s_{7}$ & $T w$ \\
\hline Coverage weight $(\mathrm{cw})$ & 2 & 2 & 2 & 2 & 2 & -1 & 1 & \\
Importance weight $(\mathrm{iw})$ & 3 & 5 & 1 & 5 & 3 & 3 & 3 & \\
Total & 6 & 10 & 2 & 10 & 6 & -3 & 3 & 34 \\
\hline
\end{tabular}

\section{Conclusions}

The health domain is a field where different people and institutions working with big data to get health services. It is necessary to access true, complete and accurate health information required to accelerate the diagnosis and treatment of a patient. Medical institutions have distributed medical records with different structures. These situation makes the interoperability and information sharing difficult even impossible.

In this work, we propose a methodology for defining working health ontologies with the aim of using them in a living health information system. To support interoperability and reuse between different health information systems, we recommend to insert related terms from the health information standards which are widely used and approved worldwide. Our methodology is also proposed to divide the domain as small as possible. Considering the health domain as a whole to model as an ontology results with 
managing problems. The methodology is demonstrated in CBLO that is developed to be used in Laboratory Information System. The proposed methodology is also evaluated by a weighted function. When the developed standards and terminologies are examined, it appears that the definitions do not contain sufficient structure and semantics with contradictions that causes problems in inferencing and reasoning. For example, in a study of the identification of ICD-10 terminology by OWL ontology, all ICD-10 codes were defined as concepts. When a new ICD-10 code is defined, the information system that uses this ontology will need to be reconfigured, which will cause the system to halt and thus cause high maintenance costs. Considering such situations, it is one of the future studies to define and develop ontology models in terms of Semantic Web technologies by evaluating terminology such as SNOMEDCT and ICD-10 widely used in the field of health domain. Designing interoperable health information systems that use defined ontologies as an information base and testing these systems with real health data in clinics are also determined as future works.

\section{Acknowledgments}

This study is a part of Scientific Research Project supported by Ege University's Scientific Research Project Committee: "Developing Blood Test Ontology for Personalized Healthcare Information System" (grant number "14MUH064").

\section{References}

Azarm, M. \& Peyton, L. (2018). An Ontology for a Patient-Centric Healthcare Interoperability Framework. 2018 IEEE/ACM International Workshop on Software Engineering in Healthcare Systems (SEHS), Gothenburg, 2018, pp. 34-41. doi: 10.1145/3194696.3194706.

Berners-Lee, T., Hendler, J. \& Lassila, O. (2001). The Semantic Web, Scientific American, 29-37.

Burgun, A., Botti, G., Fieschi, M. \& Le Beux, P. (1999). Sharing knowledge in medicine: semantic and ontologic facets of medical concepts. In Systems, Man, and Cybernetics, 1999. IEEE SMC'99 Conference Proceedings. 1999 IEEE International Conference on (Vol. 6, pp. 300-305). IEEE. doi: 10.1109/ICSMC.1999.816568.

Ceusters, W., Smith, B. \& De Moor, G. (2005). Ontology-based integration of medical coding systems and electronic patient records. MIE2005. doi:10.1.1.472.1489.

Corcho, O., Fernández-López, M. \& Gómez-Pérez, A. (2003). Methodologies, tools and languages for building ontologies. Where is their meeting point? Data \& knowledge engineering, 46(1), 4164. doi: 10.1016/S0169-023X(02)00195-7.

Cristani, M. \& Cuel, R. (2005). A survey on ontology creation methodologies. International Journal on Semantic Web and Information Systems (IJSWIS), 1(2), 49-69. doi: 10.4018/978-1-59904-4262.ch004.

Del Mar Roldán-Garcia, M., Garcia-Godoy, M. J., Aldana-Montes, J. F. (2016). Dione: An OWL representation of ICD-10-CM for classifying patients' diseases. Journal of Biomedical Semantics, 7(1), 62. doi:10.1186/s13326-016-0105-x.

Dorn, R. \& Jager, F. (2004). Semia: semi-automatic interactive graphic editing tool to annotate ambulatory ECG records. Computer methods and programs in biomedicine, 75 (3): 235-249. doi: 10.1016/j.cmpb.2004.02.005

Edinger, T., Cohen, A. M., Bedrick, S., Ambert, K. \& Hersh, W. (2012). Barriers to Retrieving Patient Information from Electronic Health Record Data: Failure Analysis from the TREC Medical Records Track, AMIA Annu Symp Proc.:180-188. [Online] https://www.ncbi.nlm.nih.gov/pubmed/23304287. Accessed:01 April 2019. 
Erickson, S. M., Wolcott, J., Corrigan J. M., Aspden, P. (Eds.) (2003). Patient safety: achieving a new standard for care. Institute of Medicine Committee on Data Standards for Patient Safety. Washington, DC, USA: National Academies Press.

FMA, Foundation Model of Anatomy, http://si.washington.edu/projects/fma, Accessed: 01 April 2019.

Focsa, Mircea Adrian, (2016). An Architectural Approach for Building Medical Ontologies. Applied Medical Informatics, 38(2), p.66-72.

Fung K.W. \& Bodenreider O. (2019). Knowledge Representation and Ontologies. In: Richesson R., Andrews J. (eds) Clinical Research Informatics. Health Informatics. Springer, Cham. doi:10.1007/978-3-319-98779-8 15.

Gesulga J. M., Berjame, A., Moquiala, K. S. \& Galido, A. (2017). Barriers to Electronic Health Record System Implementation and Information Systems Resources: A Structured

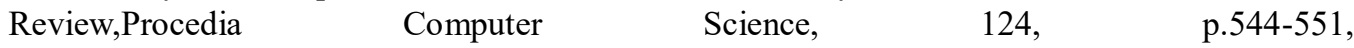
https://doi.org/10.1016/j.procs.2017.12.188.

Gómez-Pérez, A., Fernandez-Lopez, M. \& Corcho, O. (2004). Ontological Engineering: with examples from the areas of Knowledge Management, e-Commerce and the Semantic Web. ( $1^{\text {st }} \mathrm{ed}$.). Springer Publishing Company, Incorporated.

Hakkarainen, S., Strasunskas, D., Hella, L., Tuxen, S. (2005). Weighted Evaluation of Ontology Building Methods. In CAiSE Short Paper Proceedings.

ICD, The International Classification of Diseases, http://www.who.int/classifications/icd/en/, Accessed: 01 April 2019.

ICD-10 PCS, International Classification of Diseases, Version 10 - Procedure Coding System, https://bioportal.bioontology.org/ontologies/ICD10PCS, Accessed: 01 April 2019.

Kolias, V. D., Stoitsis, J., Golemati, S. \& Nikita, K. S. (2014). Utilizing Semantic Web Technologies in Healthcare. Concepts and Trends in Healthcare Information Systems, Springer International Publishing, p. 9-19. doi: 10.1007/978-3-319-06844-2_2.

Krogstie, John, (1995). Conceptual modeling for computerized information systems support in organizations. Norges Tekniske Høgskole, Institutt for Datateknikk of Telematikk. [Online] https://www.idi.ntnu.no/grupper/su/publ/phd/krogstie-thesis-electronic.pdf, Accessed: 01 April 2019.

Kruse, C. S., Kristof, C., Jones, B., Mitchell, E. \& Martinez, A. (2016). Barriers to Electronic Health Record Adoption: A Systematic Literature Review. Journal of medical systems, 40(12), 252. doi: 10.1016/j.procs.2017.12.188.

Mejino, J. L., Rubin, D. L. \& Brinkley, J. F. (2008) FMA-RadLex: An application ontology of radiological anatomy derived from the foundational model of anatomy reference ontology. Annual Symposium proceedings/AMIA Symposium. AMIA Symposium, pp. 465-469. [Online] https://www.ncbi.nlm.nih.gov/pmc/articles/PMC2656009/.

Möller, M., Daniel. S. \& Patrick, E. (2010). Modeling the international classification of diseases (ICD-10) in OWL. In International Joint Conference on Knowledge Discovery, Knowledge Engineering, and Knowledge Management, pp. 226-240. Springer Berlin Heidelberg. doi: 10.1007/978-3-642-29764-9 16. 
Nolan, N. B., Mejino, J. L. V., Detwiler, L. T., Nilsen, T. T., Martone, M. E., Turner, J. A., Rubin, D. L. \& Brinkley, J. F. (2014). Neuroanatomical domain of the foundational model of anatomy ontology. Journal of biomedical semantics, 5:(1). doi:10.1186/2041-1480-5-1.

Noy, N. F. \& McGuinness, D. L. (2001). Ontology development 101: A guide to creating your first ontology, [Online] http://www.ksl.stanford.edu/people/dlm/papers/ontology-tutorial-noymcguinness.pdf, Accessed: 01 April 2019.

Nyasulu, P. S., Paszko, C. \& Mbelle, N. (2014). A narrative review of the laboratory information system and its role in antimicrobial resistance surveillance in south Africa. Advances in Microbiology, 2014 (4), p.692-696. doi:10.4236/aim.2014.410074.

RadLex, Radiology Lexicon, http:// www.radlex.org/, Accessed: 01 April 2019.

Rajbhandari, P., Gosai, R., Shah, R. C. \& Kc, P. (2012). Semantic web in medical information systems. International Journal of Advances in Engineering and Technology, 5: 536-543. [Online] http://www.e-ijaet.org/media/53I11-IJAET1111227-MEDICAL-INFORMATION.pdf.

Schulz, S., Hanser, S., Hahn, U. \& Rogers, J. (2006). The Semantics of Procedures and Diseases in SNOMED ${ }^{\circledR}$ CT. Methods of information in medicine, 45(4), 354-358. doi: 10.1055/s-00381634088 .

Schulz S., Stegwee R. \& Chronaki C. (2019). Standards in Healthcare Data. In: Kubben P., Dumontier M., Dekker A. (eds) Fundamentals of Clinical Data Science. Springer, Cham. doi:10.1007/9783-319-99713-1_3.

Seifert, S., Kelm, M., Moeller, M., Mukherjee, S., Cavallaro, A., Huber, M. \& Comaniciu, D. (2010). Semantic annotation of medical images. SPIE Medical Imaging, pp. 762- 808. doi: $10.1117 / 12.844207$.

Sepulveda, J. L. \& Young, D. S. (2013). The ideal laboratory information system. Archives of Pathology and Laboratory Medicine, 137(8), pp.1129-1140. doi: 10.5858/arpa.2012-0362-RA.

Shridevi S., Viswanathan V. \& Saleena B. (2018). Ontology-Driven Decision Support Systems for Health Care. In: Margret Anouncia S., Wiil U. (eds) Knowledge Computing and its Applications. Springer, Singapore. doi:10.1007/978-981-10-8258-0_4.

SNOMED-CT, Systemized Nomenclature of Medicine-Clinical Terms, https//www.nlm.nih.gov/healthit/snomedct/, Accessed: 01 April 2019.

Stearns, M. Q., Price, C., Spackman, K. A. \& Wang, A. Y. (2001). SNOMED clinical terms: overview of the development process and project status. Proceedings of the AMIA Symposium, 662-666.

Tagger, Benn, (2011). An Introduction and Guide to Successfully Implementing a LIMS (Laboratory Information Management System), [Online] http://www0.cs.ucl.ac.uk/staff/B.Tagger/LimsPaper.pdf, Accessed: 01 April 2019.

Tudorache, T., Nyulas, C. I., Noy, N. F. \& Musen, M. A. (2013). Using Semantic web in ICD-11: three years down the road. International Semantic Web Conference, pp. 195-211, Springer Berlin Heidelberg. doi: 10.1007/978-3-642-41338-4_13.

UMLS, The Unified Medical Language System, https://www.nlm.nih.gov/research/umls/, Accessed: 01 April 2019.

Uschold, M. \& Gruninger, M. (1996). Ontologies: Principles, methods and applications. The knowledge engineering review, 11(02), 93-136. 